\title{
ANÁLISIS DE LAS CONCEPCIONES DE DOCENTES SOBRE LA ENSEÑANZA DE LA INGENIERÍA A TRAVÉS DE UN CUESTIONARIO DE DILEMAS Y ENTREVISTAS SEMIESTRUCTURADAS
}

\author{
ANALYSIS OF TEACHERS' CONCEPTIONS OF ENGINEERING EDUCATION \\ THROUGH A DILEMMA QUESTIONNAIRE AND SEMISTRUCTURED \\ INTERVIEWS
}

\author{
Fabián Buffa \\ María B. García \\ Marcela Natal \\ Máximo Menna \\ Lucrecia E. Moro (*) \\ Universidad Nacional de Mar del Plata \\ Argentina.
}

\section{Resumen}

El estudio de las concepciones que poseen los docentes acerca de la enseñanza es cada vez más relevante, y no muy desarrollado en carreras de ingeniería. En este trabajo se describen y comparan las concepciones sobre la enseñanza que poseen docentes de Ingeniería de la Universidad Nacional de Mar del Plata. Se realizó un estudio descriptivo con diseño ex post facto, sobre una muestra de 98 docentes. La variable en estudio fue analizada en dos dimensiones: ¿qué se enseña? y ¿cómo se enseña? Para la recolección de los datos se empleó un cuestionario de dilemas y se amplió con la realización de entrevistas. Los resultados muestran que las concepciones se encuentran distribuidas desde una postura más tradicional, centrada en los contenidos, hasta una posición más innovadora, centrada en el estudiante, concluyendo que hay una inconsistencia entre la intencionalidad sobre el qué enseñar y el qué y cómo evaluar.

Palabras clave: Docentes; ingeniería; creencias; enseñanza; cuestionario.

\section{Abstract}

$\left.{ }^{*}\right)$ Autor para correspondencia:

\section{Lucrecia E. Moro}

Facultad de Cs. Exactas y Naturales

Universidad Nacional de Mar del Plata,

Buenos Aires, Argentina.

AYL Mar del Plata AR, Deán Funes 3350,

B7602, Argentina

Correo de contacto:

lucreciamoro@gmail.com

(C)2010, Perspectiva Educacional

Http://www.perspectivaeducacional.cl

RECIBIDO: 09.10.2020

ACEPTADO: 02.07.2021

DOI: 10.4151/07189729-Vol.60-Iss.2-Art.1173
The study of the conceptions teachers have about teaching is becoming increasingly relevant; however, it has not been developed extensively in engineering programs. This paper aims to describe and compare the conceptions about teaching of professors at the Faculty of Engineering at University of Mar del Plata (UNMdP). A descriptive research with ex post facto design was carried out on a sample of 98 teachers. The variable under examination was the conception of education and this was analyzed in two dimensions: What is taught? and How is it taught? A dilemma questionnaire was used for data collection and was expanded by conducting interviews. The results obtained reveal that the conceptions are distributed from traditional, content-centred positions to a more innovative, student-centred position. It is concluded that there is inconsistency between the intentionality about what to teach and what and how to evaluate.

Keywords: professors, engineering, beliefs, teaching, questionnaire 


\section{Introducción}

Las nuevas propuestas educativas desarrolladas en los últimos años para la educación superior son esperanzadoras, en tanto recogen los resultados de las investigaciones realizadas en el campo de la didáctica de las ciencias. En el caso de las carreras de ingeniería, se propone un modelo educativo centrado en competencias, requiriendo estrategias de enseñanza que promuevan la actividad cognitiva de los estudiantes con el fin de construir el conocimiento profesional alejándose de las propuestas con cierto carácter enciclopedista, vigentes hasta la actualidad (Consejo Federal de Decanos de Ingeniería [CONFEDI], 2018; Cukierman, 2018; Kowalski, Erck \& Enríquez, 2018). Sin embargo, esta forma de entender el aprendizaje en la educación superior se encuentra muchas veces alejada de las concepciones pedagógicas de los profesores y estudiantes universitarios (Alves \& Pozo, 2020; Borgovello, Sartori \& Sanjurjo, 2018; García, 2009; García, Martín \& Mateos Sanz, 2018; Oliver, Villalonga, García, Natal \& Chaparro, 2017; Ravanal, Camacho, Escobar \& Jara, 2014), que son las que finalmente operan en la práctica (Pozo, Loo \& Martín, 2016). Si bien comienzan a observarse algunos movimientos de cambio, aún falta para ver plasmados en el aula universitaria "real" los resultados obtenidos en las aulas "ideales" de los investigadores (Mainer Baqué, 2019). En función de lo expuesto, uno de los puntos de partida fundamentales para promover estos cambios reside en indagar y poner sobre relieve las concepciones de los docentes, de manera tal que las conozcan y sean capaces de reflexionar críticamente sobre ellas a la luz de las nuevas teorías didácticas.

El problema de las concepciones docentes viene siendo profusamente estudiado en las últimas décadas, como lo prueban los trabajos de revisión de estudios que existen en torno a este tema compilados en publicaciones como las de Savasci-Acikalin (2009), Schraw (2013), SolerContreras, Cárdenas-Salgado, Hernández-Pina y Monroy-Hernández (2017) y Soler, Cárdenas y Hernández-Pina (2018). Una de las conclusiones del trabajo de revisión de 179 estudios realizado por Schraw (2013), dentro de los seis aspectos que destaca, es que muchos de ellos subutilizan el tipo de mediciones que emplean para estudiar creencias, señalando la necesidad de utilizar instrumentos que comprometan de otra manera a los participantes, como por ejemplo cuestionarios de tipo Q Sort, mapas, portafolios, entre otros. La decisión de utilizar dilemas busca ser un aporte en este sentido. De la revisión realizada por Soler-Contreras et al. (2017) y Soler et al. (2018), se recupera la idea de que las concepciones sobre la enseñanza pueden agruparse en torno a tres grandes enfoques: uno centrado en el profesor, otro centrado en el estudiante y otro centrado en la interacción profesor-estudiante, que las categorías extraídas dentro de estos enfoques tienen un doble carácter jerárquico e inclusor y que cuatro años más tarde de la revisión de Schraw (2013) siguen siendo escasos los instrumentos que van más allá de una escala Likert. Por último, del análisis realizado por 
Savasci-Acikalin (2009) se abordará el problema asumiendo que las creencias no pueden ser observadas o medidas directamente, pero deben deducirse de lo que la gente dice o pretende. Otra cuestión que surge de la revisión es que, si bien gran parte de los trabajos realizados sugieren la influencia del dominio de formación de los docentes en las concepciones sobre la enseñanza, notamos que aún falta profundizar en:

- qué contenido tienen las concepciones en el caso de los docentes que trabajan en facultades de ingeniería, ya que solo se han encontrado referencias acerca de docentes de Ciencias de una facultad de ciencias exactas y naturales (por ejemplo, los de Kramer, 2016; Martín, Vilanova \& García, 2013; Oliver et al., 2017; Vilanova, Mateos Sanz \& García, 2011).

- qué ocurre con la influencia del dominio de formación en las concepciones de docentes universitarios, particularmente cuando las disciplinas que se contrastan pertenecen todas al área de las ciencias exactas y naturales, pero tienen características diferentes. Los trabajos realizados comparan disciplinas "duras" vs. "blandas" (por ejemplo, Schommer-Aikins, Duell \& Barker, 2003), o ciencias sociales vs. ciencias naturales (por ejemplo, Norton, Richardson, Hartley, Newstead \& Mayes, 2005).

- cómo intervienen en las concepciones, si es que así sucede, otras características académicas de los docentes universitarios como, por ejemplo, la dedicación a la investigación y el ciclo de la carrera en la que trabajan. Por un lado, el hecho de estar desempeñándose como investigador podría influir, dado que forman parte de su autobiografía profesional rasgos como una profunda dedicación a la investigación que muchas veces se acompaña por una desvalorización de la tarea docente $y$, como consecuencia, una falta de interés por la reflexión acerca de las estrategias y métodos empleados; y por otro lado, el ciclo de la carrera en el que se desempeñan puede también influir ya que los estudiantes cuentan con diferentes herramientas de autorregulación de su propio aprendizaje y además las asignaturas en los ciclos superiores suelen ser más complejas y abordar contenidos más cercanos a la actividad profesional.

La investigación que se presenta en este trabajo busca realizar un aporte estudiando las concepciones sobre la enseñanza en profesores de carreras de ingeniería, grupo escasamente estudiado hasta el momento. Se las aborda desde un enfoque cuantitativo asumiendo que poseen cierto carácter implícito y se las caracteriza en relación al dominio de formación de los 
docentes y a otras características académicas como lo son la dedicación a la investigación y el ciclo de la carrera en el que desempeña su tarea.

Como perspectiva teórica para abordar el problema se entiende al profesor universitario como un profesional que va conformando su experticia y, con ella, sus concepciones sobre la enseñanza, desde una idea de progresión y continuidad a lo largo de su vida. Comienza construyendo sus creencias sobre una buena enseñanza a partir de su propia biografía como estudiante y, más tarde, desde su desempeño profesional. Según esta perspectiva, las concepciones se van conformando y eventualmente modificando, como resultado de un proceso evolutivo, filogenético y de redescripción representacional que se ve influenciado por el contexto (Errázuriz-Cruz, 2020). Si bien es evidente que el conocimiento profesional de un docente resulta de una yuxtaposición de saberes que implican diferentes grados de apropiación y explicitación (Pecharromán, 2003), se parte de la hipótesis que las manifestaciones que de dicho conocimiento aparecen en contextos educativos están guiadas fundamentalmente por las representaciones de carácter más implícito (Pecharromán, 2003; Rodrigo, Rodríguez \& Marrero, 1993) y, por lo tanto, tienen cierta dificultad para ser estudiadas.

Con respecto al contenido de las concepciones, los estudios realizados desde la perspectiva fenomenográfica han permitido crear abundante conocimiento sobre las diferentes posturas acerca de la enseñanza mantenidas por los docentes universitarios (Alves \& Pozo, 2020). Una de las conclusiones más relevantes es que las mismas se pueden agrupar en tres grandes orientaciones: por un lado, las que entienden que el profesor desarrolla su enseñanza centrándose en la materia de estudio, con el objetivo de transmitir información (tradicional); por otro, las concepciones que centran la enseñanza en el estudiante con el objetivo de ayudarlo a complejizar la comprensión que este tiene del mundo que lo rodea (innovadora); y por último, una orientación intermedia entre ambas (Ravanal et al., 2014). Dentro de cada una de estas orientaciones existen a su vez posiciones variadas, cuya descripción depende de los marcos teóricos y metodológicos desde los que se ha abordado el problema. Categorías similares también pudieron ser construidas por los autores del presente trabajo en las investigaciones realizadas en los últimos dos años, con una muestra reducida de la población actual a través de entrevistas individuales a docentes de Ingeniería (Buffa, Moro, Massa, García \& Fanovich, 2018). En la Tabla 1 se presenta una breve descripción de las características distintivas de cada una de las tres concepciones principales. 
Tabla 1

Principales concepciones encontradas en docentes universitarios

\begin{tabular}{|c|c|c|}
\hline $\begin{array}{l}\text { A. Concepción centrada en el } \\
\text { profesor (tradicional) }\end{array}$ & B. Concepción Intermedia & $\begin{array}{l}\text { C. Concepción centrada en el } \\
\text { estudiante (innovadora) }\end{array}$ \\
\hline $\begin{array}{l}\text { Transmisión de } \\
\text { conocimientos de forma }\end{array}$ & $\begin{array}{l}\text { Interacción profesor- } \\
\text { estudiante. }\end{array}$ & $\begin{array}{l}\text { Cambio conceptual y } \\
\text { desarrollo intelectual. }\end{array}$ \\
\hline $\begin{array}{l}\text { estructurada. } \\
\text { El propósito es presentar la } \\
\text { asignatura en forma más o } \\
\text { menos estructurada para } \\
\text { facilitar al estudiante el } \\
\text { proceso de asimilación de los } \\
\text { contenidos. } \\
\text { Al estudiante se lo ve como } \\
\text { un receptor pasivo. }\end{array}$ & $\begin{array}{l}\text { Se caracteriza por la } \\
\text { relevancia que se otorga a la } \\
\text { interacción profesor- } \\
\text { estudiante. Se hace hincapié } \\
\text { en las actividades de } \\
\text { aprendizaje tales como } \\
\text { experimentos, o la resolución } \\
\text { de problemas. }\end{array}$ & $\begin{array}{l}\text { El protagonista es el } \\
\text { estudiante. El papel del } \\
\text { profesor es ayudar al } \\
\text { estudiante a aprender, } \\
\text { confrontando lo que sucede a } \\
\text { su alrededor con lo que } \\
\text { piensa. }\end{array}$ \\
\hline
\end{tabular}

Fuente: Elaboración propia.

Otro resultado obtenido como consecuencia del relevamiento de estudios previos de otros investigadores y propios (por ejemplo, García, 2009; Pacheco Lora, 2019; Ramsden, Prosser, Trigwell \& Martin, 2007), sugieren que las concepciones de los docentes cambian al consultarlos respecto de qué se enseña y luego hacerlo respecto de cómo se enseña. En función de estos antecedentes, se estudiarán las concepciones de carácter más bien implícito que poseen los docentes que se desempeñan en carreras de ingeniería respecto de qué se enseña (qué contenidos) y cómo se enseña (qué estrategias). Y se indagarán posibles relaciones entre estas concepciones y aspectos académicos de los sujetos participantes, como título de grado, rama de la ingeniería a la que pertenece, cargo en la universidad, dedicación, etc.

\section{Metodología}

Como continuidad del estudio fenomenográfico realizado previamente por Buffa et al. (2018), en el presente trabajo se profundizó el análisis desde un enfoque cuantitativo. Se realizó un estudio descriptivo de las concepciones de los docentes utilizando un cuestionario de dilemas y se complementó esta información con entrevistas semiestructuradas en profundidad.

\subsection{Participantes}

Participaron de este estudio 98 profesores universitarios que se desempeñan como docentes en la Facultad de Ingeniería de la Universidad Nacional de Mar del Plata. A fin de que la muestra fuera tan homogénea como fuese posible, participaron docentes de las diez carreras 
ANÁLISIS DE LAS CONCEPCIONES DE DOCENTES SOBRE LA ENSEÑANZA DE LA INGENIERÍA A TRAVÉS

DE UN CUESTIONARIO DE DILEMAS Y ENTREVISTAS SEMIESTRUCTURADAS

de grado en ingeniería que se dictan en la facultad, tanto del ciclo básico como del ciclo superior.

\subsection{Diseño de investigación y tipo de estudio}

Se utilizó un diseño ex post facto prospectivo simple debido a que las variables en estudio ya han tomado sus valores en el momento en que se inicia la investigación.

Se tuvieron en cuenta las siguientes variables:

V1: concepciones de los docentes sobre la enseñanza.

V2: características académicas de los docentes participantes.

Las categorías y dimensiones de las variables V1 y V2 se muestran en las Tablas 2 y 3.

\section{Tabla 2}

Dimensiones y categorías de la variable V1

\begin{tabular}{lll}
\hline Variable & Dimensiones & Categorías \\
\hline & & $\begin{array}{l}\text { A. Concepción centrada en el docente con } \\
\text { énfasis en los contenidos (tradicional) [1] }\end{array}$ \\
\cline { 3 - 3 } $\begin{array}{l}\text { Concepciones sobre la } \\
\text { enseñanza }\end{array}$ & ¿Qué se enseña? & ¿. Concepción intermedia [2]
\end{tabular}

Fuente: Elaboración propia.

La división de la dimensión cómo se enseña en las dos subdimensiones indicadas, obedece a resultados encontrados por los autores y otros investigadores que muestran que las concepciones varían al modificarse el contexto entendido como tema o aspecto escogido para la indagación (contenido o evaluación) (Buffa, Massa, Moro \& García, 2019; Oliver et al., 2017).

\section{Tabla 3}

Características académicas: variables y categorías de la variable V2

\begin{tabular}{ll}
\hline Variables & Categorías \\
\hline V2(A) - Título profesional & $\begin{array}{l}\text { ingeniero } \\
\text { licenciado } \\
\text { profesor }\end{array}$ \\
\hline V2(B) - Dedicación de su cargo & $\begin{array}{l}\text { simple (docencia) } \\
\text { parcial (docencia e investigación - extensión) } \\
\text { exclusiva (docencia e investigación - extensión) }\end{array}$
\end{tabular}


Fuente: Elaboración propia.

\subsection{Instrumentos}

Para poder tener en cuenta la perspectiva teórica adoptada y, a su vez, consultar a un número representativo de docentes, se optó por utilizar: a) un cuestionario de dilemas, el cual se complementó con b) entrevistas semiestructuradas.

Los dilemas, a diferencia de otro tipo de instrumentos, como por ejemplo las escalas Likert, obligan a decantar las posiciones de forma más clara. Si bien es cierto que en este tipo de instrumentos todas las alternativas hacen afirmaciones que pueden considerarse acertadas, el profesor tiene que inclinarse finalmente por una: aquella que, sin ser con seguridad la que reúne todos los matices que podrían reflejarse en una respuesta producida por el propio docente, se aproxima mejor a su concepción del problema planteado. Por otra parte, los dilemas tienen carácter argumentativo, es decir, una misma posición puede defenderse por razones diferentes. Por último, permiten contextualizar las respuestas, lo que estaría en línea con el marco teórico de las teorías implícitas que destaca su carácter contextual. En síntesis, los cuestionarios de dilemas constituyen una opción válida que permite el acceso a diferentes niveles representacionales y resuelven el problema de la representatividad de la muestra.

\subsubsection{Cuestionario de dilemas}

El cuestionario consta de 12 dilemas, cada uno de ellos con tres opciones de respuesta, una para cada una de las concepciones encontradas en el estudio fenomenográfico anterior (Buffa et al., 2018) y que constituyen las categorías de la variable V1 en el estudio actual (1, 2 y 3, de acuerdo a las categorías enunciadas en la Tabla 2, de las cuales el sujeto debía escoger solo una opción). En el encabezado, el docente indica su título, la dedicación de su cargo y las asignaturas en las que participa (variable V2, Tabla 3). El instrumento fue sometido a un proceso de validación que incluyó la validez de contenido, la revisión de la claridad, la validez de constructo y la consistencia interna del instrumento (Alfa de Cronbach=0,796) (Buffa et al., 2020).

La Tabla 4 agrupa los dilemas de acuerdo a la dimensión del análisis que evalúan. 
ANÁLISIS DE LAS CONCEPCIONES DE DOCENTES SOBRE LA ENSEÑANZA DE LA INGENIERÍA A TRAVÉS

DE UN CUESTIONARIO DE DILEMAS Y ENTREVISTAS SEMIESTRUCTURADAS

Tabla 4

Dilemas que evalúa cada dimensión de la variable V1

\begin{tabular}{lll}
\hline \multicolumn{2}{l}{ Dimensión } & Dilemas \\
\hline ¿Qué se enseña? & D2, D5, D8, D9 \\
\hline \multirow{2}{*}{ ¿Cómo se enseña? } & Función y estrategias & D1, D4, D6 \\
\cline { 2 - 3 } & Evaluación & D3, D7, D10, D11, D12 \\
\hline
\end{tabular}

Fuente: Elaboración propia.

\subsubsection{Entrevistas semiestructuradas}

El protocolo para entrevistar a individuos seleccionados, está conformado por un cuestionario de 23 preguntas semiestructuradas vinculadas a diferentes aspectos de la enseñanza que incluyen las dimensiones de la variable V1, diseñado por Buffa et al. (2018). A modo de ejemplo, se presentan algunas de ellas: ¿Qué es lo que fundamentalmente aprenden los estudiantes en su asignatura? ¿Qué recursos utiliza para atender a la diversidad de alumnos que probablemente tiene en su clase? ¿Cómo se da cuenta de que el alumno aprendió? ¿Qué información de sus estudiantes considera importante para planificar su asignatura?

\subsection{Procedimiento de recolección y análisis de datos}

La recolección y análisis de dato se llevó a cabo en dos etapas:

a) Primera etapa: el cuestionario de dilemas fue suministrado 1 a 1 a los 98 docentes que formaron parte de este estudio, quienes contaron con tiempo suficiente para responderlo y entregarlo a uno de los autores de este trabajo. A partir de las respuestas dadas en este cuestionario, se realizó una distribución de frecuencias para cada dimensión y subdimensión estudiada. A continuación, para estudiar las relaciones de interdependencia entre variables y conocer cómo está estructurada esta relación se efectuó un análisis factorial de correspondencias múltiples (AFCM). Para obtener una partición del conjunto de datos y una caracterización de cada una de las clases se aplicó una clasificación jerárquica con el método de agregación alrededor de centros móviles (K-medias), logrando obtener clases homogéneas bien diferenciadas entre sí. La aplicación de esta metodología se realizó utilizando el lenguaje R, con el paquete de análisis multivariado en R FactoMineR ${ }^{\circledR}$ (Husson, Lê \& Pagès, 2017).

b) Segunda etapa: con el objetivo de confirmar y describir mejor cada una de las clases, se complementaron los resultados con dos entrevistas semiestructuradas, cada una de ellas con 
ANÁLISIS DE LAS CONCEPCIONES DE DOCENTES SOBRE LA ENSEÑANZA DE LA INGENIERÍA A TRAVÉS

DE UN CUESTIONARIO DE DILEMAS Y ENTREVISTAS SEMIESTRUCTURADAS

un docente representativo (sujeto icónico de cada clase, seleccionado a partir del estudio estadístico). Las mismas se registraron en formato digital y duraron aproximadamente una hora. Se llevaron a cabo en un marco natural, abierto y en tono de conversación, para permitir que la posición de los entrevistados emergiera de manera espontánea. Se analizó el conjunto de las respuestas dadas por los sujetos seleccionados.

\section{Resultados}

Se presenta, en primer lugar, una descripción de las concepciones sobre la enseñanza encontradas en los docentes que formaron parte de este trabajo a partir de la distribución de frecuencias para las distintas categorías de respuesta a cada uno de los dilemas, las que se relacionan con las diferentes categorías de la variable V1, mostradas en la Tabla 2. Luego, se presentan los resultados encontrados de la correlación entre estas concepciones y algunas características académicas de los docentes, mostradas en la Tabla 3. Y para confirmar estos últimos resultados, se muestran algunas respuestas significativas de dos docentes tipo de las clases halladas, a preguntas realizadas a través de una entrevista semiestructurada.

\subsection{Descripción de las concepciones}

En la Figura 1 se presentan los resultados correspondientes a la distribución de frecuencias para cada categoría de respuesta (tradicional [1], intermedia [2] o innovadora [3]), en el grupo de dilemas que conformaron cada dimensión de la variable "Concepciones sobre la enseñanza" de los docentes de Ingeniería (¿qué se enseña? y ¿cómo se enseña?, esta última analizada en las dos subdimensiones ya descritas). 


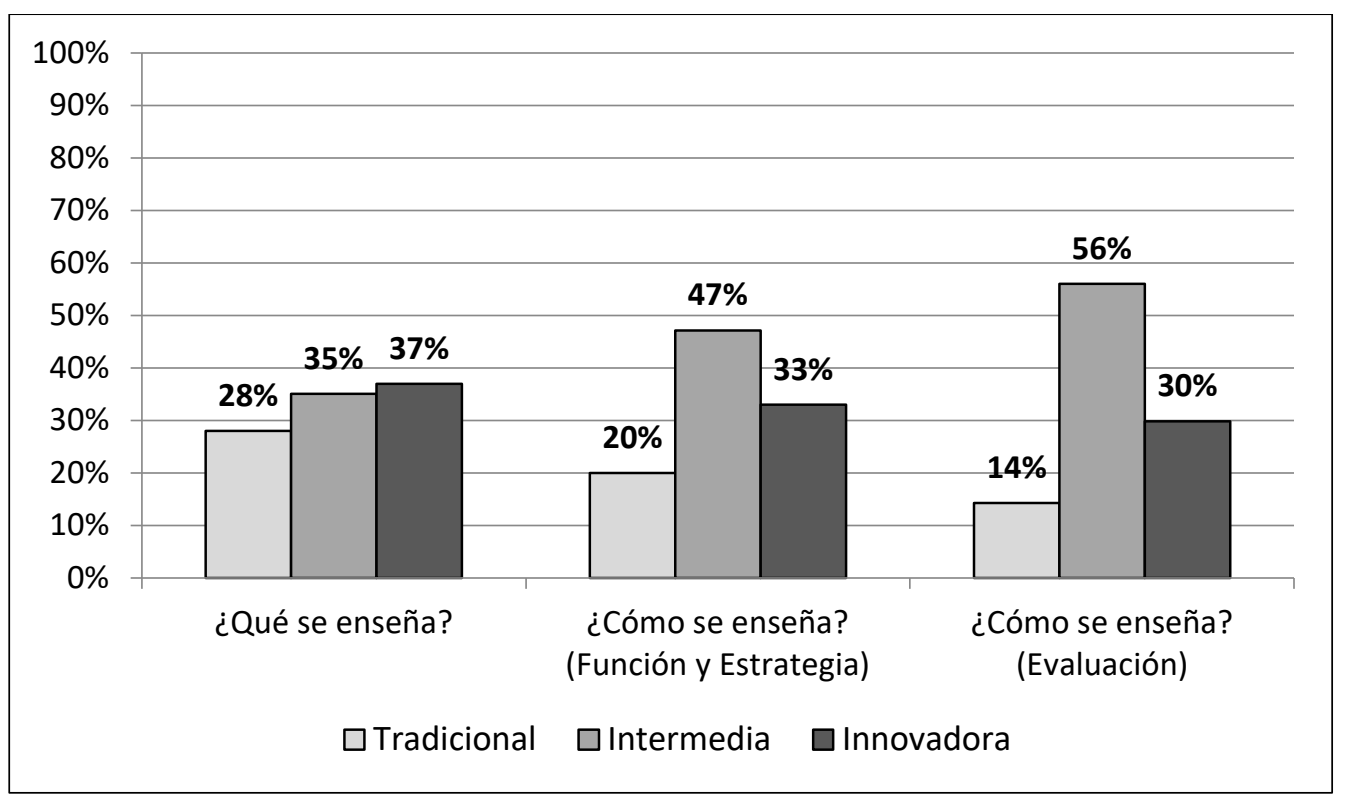

Figura 1: Concepciones de docentes por dimensión. Distribución de frecuencias en porcentajes.

Fuente: Datos de la investigación.

Se puede observar, en la dimensión ¿qué se enseña?, una distribución semejante entre la posición intermedia e innovadora, las que no se diferencian demasiado de la tradicional. Por ejemplo, frente al siguiente dilema:

En relación con la extensión de los programas de las asignaturas, algunos docentes piensan que se debe:

a) Seleccionar los contenidos más adecuados para que los estudiantes razonen y desarrollen estrategias de aprendizaje autónomo, aunque no se complete el programa.

b) Enseñar los contenidos que surgen de la lógica disciplinar, ya que son indispensables para que el estudiante avance en la carrera.

c) Enseñar todos los contenidos que surgen de la lógica de la disciplina que se puedan llegar a dar, cuidando que los estudiantes razonen y comprendan lo más posible.

27 de los docentes participantes eligieron la respuesta (b), que muestra una predominancia de los contenidos sobre cualquier otra consideración (1-tradicional), 36 eligieron la (c), donde los contenidos siguen siendo relevantes, pero se atiende también a la respuesta de los estudiantes (2-intermedia), y 35 optaron por la (a), en la que predomina el desarrollo de una competencia en el estudiante por sobre los contenidos enseñados (3-innovadora). 
Cuando se analiza la dimensión ¿cómo se enseña?, el predominio de la posición intermedia pasa a ser dominante, aún más en los aspectos relacionados con la evaluación. Así, frente al dilema:

Un tema de discusión recurrente es la actitud que deben tener frente a las preguntas que los estudiantes hacen durante los exámenes. Algunos piensan que:

a) Se deben aclarar las reglas previamente, y no responder ninguna pregunta durante el examen, porque la comprensión de las consignas es parte de la evaluación y porque es la mejor manera de asegurarse de ser justos.

b) Hay que contestar individuamente porque las dudas que se pueden presentar son propias de cada estudiante.

c) Se pueden contestar solo preguntas de enunciado en forma general y en voz alta para que todos escuchen lo mismo.

9 docentes seleccionaron la opción (a), la que no tiene en cuenta al estudiante y las posibles dudas que se le puedan presentar en una situación de examen, amparándose en un concepto subjetivo de justicia (1-tradicional); 56 docentes eligieron la opción (c), que si bien considera la respuesta a las dudas, uniformiza al grupo de estudiantes y no tiene en cuenta las necesidades de cada uno (2-intermedia), lo que sí es tenido en cuenta en la consigna (b) (3-innovadora), opción que fue la preferida por 28 docentes. 5 docentes no respondieron este dilema o señalaron más de una alternativa.

O frente a este otro dilema:

\section{Respecto de cómo hacer preguntas para evaluar:}

a) Las preguntas deben ser lo más concretas y claras posible, como para que los alumnos no se dispersen en las respuestas.

b) Las preguntas deben ser lo más concretas y claras posibles, pero a su vez permitirle al alumno llegar a la misma respuesta por distintos caminos.

c) Las preguntas deben ser lo suficientemente abiertas como para que cada alumno pueda organizar su propia respuesta.

Aquí se encontraron respuestas que confirman el perfil del grupo en esta dimensión: 10 docentes eligieron la respuesta (a), que se dirige a construir preguntas de respuesta cerrada, que conduzcan a que todos los estudiantes respondan de una única manera (1-tradicional), 69 
optaron por la respuesta (b), que deja abierta la posibilidad a que los estudiantes elijan distintos caminos para alcanzar la misma respuesta (2-intermedia), y los otros 12 se inclinaron por la (c), que pone énfasis en el camino desarrollado por el estudiante para alcanzar la respuesta por sobre el resultado (3-innovadora). 7 docentes no respondieron este dilema 0 señalaron más de una alternativa.

\subsection{Análisis de las relaciones entre las concepciones y algunas características académicas de los docentes participantes}

Para continuar profundizando el análisis y abordar el segundo objetivo, se indagaron posibles relaciones entre las concepciones de los docentes y algunas de sus características académicas, tales como el título profesional, la dedicación a la investigación y el ciclo en que se desempeña (ver Tabla 3).

Se desarrolló un estudio estadístico del conjunto de datos que comprendió las respuestas de los 98 sujetos que compusieron la muestra y las características académicas de cada sujeto. Se realizó un análisis factorial de correspondencias múltiples (ACM), tal como se indicó en el apartado correspondiente a los procedimientos y un análisis de conglomerados (clústeres). EI conjunto de dilemas, sus categorías y las características académicas de los docentes fueron consideradas variables activas. Las respuestas obtenidas por medio del cuestionario se agruparon en conjuntos homogéneos (clases). A continuación se describen las clases con mayor porcentaje de individuos (Tablas 5 y 6 ).

Tabla 5

Caracterización de la clase 1 (46\%) 45 individuos

\begin{tabular}{lll}
\hline Modalidad & Clase/Modalidad ${ }^{*}(\%)$ & Modalidad/Clase ${ }^{* *}(\%)$ \\
\hline D8=1 & 82.1 & 82.0 \\
\hline D9=1 & 91.3 & 53.8 \\
\hline$D 7=1$ & 75.9 & 56.4 \\
\hline$D 1=1$ & 68.6 & 61.5 \\
\hline$D 4=2$ & 69.0 & 51.3 \\
\hline$D 2=2$ & 63.6 & 53.8 \\
\hline$D 5=2$ & 61.1 & 56.4 \\
\hline$D 3=2$ & 57.1 & 61.5 \\
\hline$D 6=1$ & 80.0 & 20.5 \\
\hline
\end{tabular}


ANÁLISIS DE LAS CONCEPCIONES DE DOCENTES SOBRE LA ENSEÑANZA DE LA INGENIERÍA A TRAVÉS

DE UN CUESTIONARIO DE DILEMAS Y ENTREVISTAS SEMIESTRUCTURADAS

\begin{tabular}{lcc}
\hline D10 $=2$ & 52.8 & 71.8 \\
\hline Título=Ingeniero & 49.3 & 87.2 \\
\hline
\end{tabular}

Fuente: Elaboración propia.

Esta clase se caracteriza por estar conformada por sujetos que eligieron las opciones 1 o 2 en 10 de los 12 dilemas en un porcentaje mayor al 50\%. Por ejemplo, en el dilema 9, el 91,3\% (*) de los docentes que respondieron en la opción 1, forma parte de esta clase. Por otro lado, también los datos muestran que el 53,8\% (**) de los docentes que conforman la clase está formado por sujetos que eligieron la opción 1 en este dilema.

En este grupo se encuentran el $49,3 \%\left(^{*}\right)$ de los ingenieros, siendo que el $87,2 \%\left({ }^{* *}\right)$ de los individuos de esta clase poseen este título.

Tabla 6

Caracterización de la Clase 2 (54\%) 53 individuos

\begin{tabular}{lll}
\hline Modalidad & Clase/Modalidad * (\%) & Modalidad/Clase ** (\%) \\
\hline D8=3 & 85.0 & 75.6 \\
\hline D4=3 & 70.9 & 86.7 \\
\hline D2=3 & 72.7 & 71.1 \\
\hline D9=2 & 68.1 & 71.1 \\
\hline D7=3 & 100.0 & 22.2 \\
\hline D1=3 & 84.2 & 35.6 \\
\hline$D 5=3$ & 72.4 & 46.7 \\
\hline$D 3=3$ & 66.7 & 57.8 \\
\hline
\end{tabular}

Fuente: Elaboración propia.

La segunda clase se caracteriza por la presencia de individuos que eligieron la opción 3 en 7 de los 8 dilemas, en un porcentaje mayor al 66\%. Por ejemplo, el $85 \%(*)$ de los que seleccionaron la opción 3 en el dilema 8 se encuentran en este grupo, mientras que el 75,6\% (**) de esta clase está formada por esos individuos.

\subsection{Ampliación del análisis mediante entrevistas}

Un abordaje complementario para confirmar y describir con más detalle las clases encontradas se realizó a partir de entrevistas semiestructuradas con el protocolo descrito en el apartado 2.3 
Instrumentos. Para el presente estudio, se seleccionaron los sujetos tipo de cada clase a los que denominaremos de aquí en adelante docente $\mathrm{A}$ (clase 1) y docente $\mathrm{B}$ (clase 2 ).

Respecto al ¿qué se enseña?, ante la pregunta "¿Qué es lo que fundamentalmente aprenden los estudiantes en su asignatura?", el docente A responde: "Tener criterio. De todos los temas ver cuáles son los conceptos más importantes". Lo conceptual cobra relevancia sobre cualquier otro aspecto de la enseñanza. La acción del estudiante es limitada a una jerarquización de los contenidos. A la misma pregunta, el docente B responde: "Aprender a utilizar una serie de herramientas [...] que le darán más sentido a lo que haga en su profesión". Lo conceptual deja lugar a lo procedimental; el estudiante pasa a estar en el centro de la ejecución de esas herramientas. La profesión está presente en el objeto y en el contexto de la enseñanza.

Respecto al ¿cómo se enseña?, y en relación a las estrategias, frente a la pregunta "¿Qué recursos utiliza para atender a la diversidad de alumnos que probablemente tiene en su clase?", el docente A responde: "Estoy permanentemente en la facultad. Ellos saben que pueden venir a hacer consultas. No advierto diferencias entre los estudiantes de las dos distintas carreras que asisten". Más allá de no distinguir diferencias, el docente espera que el estudiante se acerque, no acude a buscarlo, no pone su mirada en él. Él no está en el centro de su ocupación. El docente B responde: "Todos los estudiantes son diferentes. Trato de escucharlos para darme cuenta de estas diferencias. Y los hago hablar y trato de sacarles ese miedo a equivocarse". El estudiante vuelve a ocupar una posición central, sus diferencias influyen en las estrategias a emplear. Este docente, en otro momento de la entrevista, interrogado acerca de qué información de sus estudiantes considera importante para planificar su asignatura, respondió: "Haría un diagnóstico para ver cómo llegaron de las asignaturas anteriores. Saber cuántas asignaturas están cursando, si trabajan, qué disponibilidad de tiempo tienen. Hay grupos muy heterogéneos: alumnos que vienen de escuelas privadas, otros de escuelas públicas... la idea es integrarlos".

Frente a la pregunta “¿Cómo se da cuenta de que el alumno aprendió?", el docente A responde: "Te vas dando cuenta cuando tienen interés en los temas". Si bien no hay referencia a las evaluaciones tradicionales, tampoco la hay al proceso de aprendizaje del estudiante. Solamente un observable, el interés del estudiante, que muchas veces no es demostrado por cuestiones que atañen a su personalidad y que, por lo tanto, no lo convierte en un insumo a tener en cuenta en todos los casos.

En cambio, el docente B frente a la misma pregunta, responde: "Los parciales nos dan una evaluación global, pero si sabe o no, no me doy cuenta en esas evaluaciones. En realidad me voy 
dando cuenta de a poco, cuando voy haciendo preguntas en las prácticas, por sus intervenciones. Hay muchas falencias en evaluar con dos o tres parciales. Sus avances los veo día a día. A veces he cambiado una nota por la devolución del estudiante, porque me di cuenta de que el error no era porque el estudiante no sabía, sino porque la pregunta no estuvo bien formulada". Nuevamente se observa la visión centrada en el estudiante; para saber qué es lo que aprendió es necesario evaluar un proceso, no son suficientes dos o tres momentos y con una evaluación objetiva. Hay un seguimiento de los avances de los estudiantes, un registro de sus intervenciones en las clases.

\section{Discusión}

\subsection{Descripción de las concepciones}

En los resultados encontrados se observa una diferencia en las concepciones de los docentes según la dimensión analizada: cuando responden a los dilemas referidos al qué se enseña, las concepciones intermedias e innovadoras reúnen el 72\%, con muy poca diferencia entre ellas. Mientras que en los dilemas que se ocupan del cómo se enseña ambas concepciones concentran más del $80 \%$, pero las diferencias entre ellas se hacen notorias en favor de la posición intermedia, aún más en los dilemas referidos a la evaluación. Es decir que, en esta dimensión, las concepciones innovadoras han cedido lugar a las de mayor contenido tradicional. Resultados similares han sido encontrados por García (2009) en docentes de Ciencias Exactas y Naturales, por Oliver et al. (2017) en docentes universitarios de Matemática, por Kramer (2016) en estudiantes del profesorado de Biología y por Martín et al. (2013) en docentes universitarios de Biología.

Esto se podría explicar si tenemos en cuenta que al referirnos al ¿qué se enseña? las concepciones transcurren en el campo de lo discursivo, de las reflexiones que el docente hace antes de entrar al aula, de aquellas que se disparan durante la planificación de su tarea. Se desplazan en el terreno de lo conceptual, donde el "deber ser" de la enseñanza se antepone a la realidad del aula. Cuando se pasa al ¿cómo se enseña? entramos al campo de la acción, de lo procedimental, de cómo opera en el aula aquella planificación. Y es en estos casos donde la ausencia de formación docente deja lugar a lo adquirido a través de la experiencia, tanto como estudiante como docente, alumbrando concepciones menos sofisticadas y más pragmáticas, más cercanas a las concepciones reales de los docentes y las que, probablemente, guíen su propia práctica tal como se menciona en el marco conceptual que sustenta el presente trabajo. Esto se hace más visible cuando se enfoca la evaluación, donde la función acreditadora (posición tradicional) parece imponerse sobre la formativa (posición innovadora). Lo anterior 
muestra que los avances en el campo de la enseñanza de las ciencias encontrados por los investigadores en los últimos años aún no se han plasmado en el aula.

\subsection{Relación entre las concepciones de los docentes y algunas características académicas}

En función de estos resultados se puede describir a las concepciones sobre la enseñanza de los docentes de la población estudiada alrededor de dos clases. Tomando como referencia las categorías descritas en el marco teórico, se observa una clase formada por aquellos sujetos que conciben la enseñanza desde una posición que resulta de una combinación entre una orientación tradicional y una intermedia, caracterizada también por los docentes cuyo título de base es el de ingeniero, y otra, en la que se encuentran los sujetos que claramente tienen una posición innovadora frente a la enseñanza. Las características académicas no son identificadoras de esta clase. Resultados previos encontrados en las investigaciones realizadas por los autores en una población considerablemente menor, pero con las mismas características (Buffa et al., 2018), indicaron que las concepciones de los docentes de Ingeniería se agrupaban principalmente en tres categorías: una tradicional, centrada en el profesor, una innovadora, centrada en el estudiante, y una intermedia. La distribución de los docentes variaba dependiendo de la dimensión analizada: equilibrada respecto al qué se enseña y claramente inclinada a la tradicional respecto al cómo se enseña. Los límites entre las categorías fueron difusos, dependiendo también de las dimensiones analizadas. En síntesis, al ampliar la muestra, se encuentran las mismas categorías pero los docentes ahora se dividen en los dos grupos antes descritos.

Si se analizan estos resultados a la luz de las dimensiones planteadas para evaluar las concepciones, los resultados son los siguientes:

Tabla 7

Concepciones de los docentes de cada clase según las dimensiones analizadas

\begin{tabular}{|c|c|c|c|c|}
\hline Dimensión & & & Clase 1 & Clase 2 \\
\hline ¿Qué se enseña? & & & Tradicional - intermedia & Innovadora \\
\hline \multirow[t]{2}{*}{ ¿Cómo se enseña? } & $\begin{array}{l}\text { Función } \\
\text { estrategias }\end{array}$ & $\mathrm{y}$ & Tradicional - intermedia & Innovadora \\
\hline & Evaluación & & Tradicional - intermedia & Innovadora \\
\hline
\end{tabular}

Fuente: Elaboración propia. 
Respecto al qué se enseña, teniendo en cuenta los dilemas que conforman esta dimensión, la clase 1 está formada por docentes que basan su desempeño en transmitir los contenidos conceptuales de la disciplina, a veces con una mirada global de la asignatura. El foco está en la comprensión de los contenidos como un todo. El profesor presenta los temas y traza vínculos entre estos y otros contenidos de la asignatura. También, propone actividades que favorezcan la reproducción del conocimiento válido. Estas actividades son fundamentalmente ejercicios y prácticas de laboratorio que se siguen a modo de receta. Aparece la figura del estudiante activo pero repetitivo.

Por otro lado, en la clase 2 se encuentran los docentes que poseen una concepción innovadora de la enseñanza, que enseñan capacidades que conducen a la autonomía del estudiante, apuntando a que los mismos internalicen, reorganicen y reconstruyan los conceptos en el proceso de aprendizaje. Estos docentes buscan que los estudiantes incorporen los contenidos a partir de los cuales se pueden interpretar, analizar y estudiar fenómenos vinculados con la asignatura en la que trabaja. Se plantean situaciones problemáticas para la búsqueda de soluciones más que ejercicios de resolución mecánica. Se busca provocar un cambio conceptual, en el sentido epistemológico general, de manera de desarrollar capacidades propias de un futuro ingeniero.

Respecto al cómo se enseña, los docentes de la clase 1 son aquellos cuyas concepciones se identifican con la idea de que se enseña transmitiendo de manera clara un conocimiento previamente seleccionado y organizado por un experto. El profesor presenta la información relacionando contenidos para facilitar al estudiante el proceso de asimilación y mantener su atención. La manera de entender la enseñanza, centrada en contenidos verbales, reserva para el docente el lugar de "explicador". El docente en este enfoque puede ser entendido como el gasolinero que llena el depósito de conocimientos del estudiante (Claxton, 1990). El conocimiento académico sigue siendo el atributo más importante de un buen profesor, pero se pone más énfasis en la calidad de la presentación.

Para los docentes agrupados en la clase 2, el centro está en el estudiante. Se involucran con el conocimiento disciplinar con la intención de ayudar a los estudiantes a aprender a aprender, a autorregular su proceso de aprendizaje. Acompañando este proceso, el profesor puede asumir una función de guía, con diferentes grados de implicación o dirección: puede comportarse como un mediador entre el conocimiento y el estudiante (Díaz \& Hernández, 2015), siendo el tutor del aprendizaje, donde cede parte de la responsabilidad al estudiante pero mantiene la dirección del proceso. 
En esta dimensión también se encuentran aspectos relacionados a la evaluación, donde las diferencias se hacen más notorias, como se observó en la Figura 1. Los docentes de la clase 1 son aquellos que tienen en cuenta a la evaluación como para calificar y acreditar los resultados. Hacen hincapié en el conocimiento memorístico y enfatizan en el producto del aprendizaje (lo observable). El desempeño de los estudiantes, sus recorridos, sus superaciones, no son tenidos en cuenta por estos docentes. El principal instrumento es el examen de lápiz y papel, y las instancias se reducen a un número acotado. Los docentes de la clase 2 siguen poniendo énfasis en la evaluación como un resultado, pero tienen en cuenta ciertos elementos del proceso. Estos docentes incorporan algunas herramientas para este seguimiento: uso de rúbricas para facilitar el monitoreo de los aprendizajes de los estudiantes, utilización de diferentes estrategias de evaluación, uso de la evaluación para la regulación del proceso de enseñanza.

Este predominio de visiones intermedias e innovadoras también fue encontrado por Vilanova et al. (2011) en una población de docentes de Ciencias (Física, Química, Biología y Matemática) y por Oliver et al. (2017) en un conjunto de docentes y estudiantes de un profesorado en Matemáticas. En ambos casos, el predominio de las visiones innovadoras se relaciona con los aspectos vinculados con el qué se aprende, mientras que en aspectos asociados con el cómo se aprende y cómo se evalúa, predominan concepciones vinculadas a concepciones intermedias.

En cuanto al aporte de las reflexiones de los docentes entrevistados, se observó que se mostraron en línea con las caracterizaciones de las concepciones descritas para cada clase. Este análisis complementario permitió, por un lado, confirmar las clases encontradas a partir del ACM y, por otro, describirlas de manera más exhaustiva.

\section{Conclusiones}

Respecto de las concepciones sobre la enseñanza de los docentes de Ingeniería, los resultados obtenidos muestran que se distribuyen en dos clases. La primera, formada fundamentalmente por ingenieros y caracterizada por concebir el proceso de enseñanza de manera similar a las acciones de consignación bancaria en la cual se deposita un conocimiento en la "mente del educando" -más o menos adaptado por un experto, dependiendo del tipo de concepción-y se extrae de la misma a través de procesos evaluativos (Flórez Nisperuza, Páez, Fernández \& Salgado, 2019). Buscan promover espacios de enseñanza que permitan que el estudiante aprenda lo que los docentes transmiten sobre un determinado tópico y se apropie formalmente de los conocimientos. Se aprende a través de un proceso de captación, atención y ejercitación de contenidos, proceso que posibilita aplicar el conocimiento adquirido, pero que difícilmente se pueda interpretar o reestructurar. La segunda clase de docentes concibe su rol como un tutor que debe ir habilitando cada vez mayor protagonismo al estudiante durante la experiencia educativa (Boud, Cohen \& Sampson, 2014). Desde este punto de vista, se enseña 
de forma tal de promover en el estudiante el desarrollo de habilidades que le permitan comprender los contenidos de modo que no solo sea capaz de aplicarlos para interpretar distintos tipos de fenómenos sino también de, eventualmente, interpelarlos con una actitud científica (Mainer Baqué, 2019). Cabe realizar una mención respecto de cierta inconsistencia encontrada en las concepciones de los docentes de Ingeniería al cambiar la dimensión de análisis. Se evidenció falta de coherencia entre lo que pretenden enseñar y luego lo que evalúan, mostrando en este último aspecto concepciones tradicionales que buscan verificar el recuerdo de información. A la luz de estos resultados se puede coincidir con lo expresado en los estudios realizados por Pérez Echeverría, Mateos, Pozo y Scheuer (2001) y Ventura (2016), diciendo que los docentes asumen un aprendizaje activo pero reproductivo, y dan cuenta de una visión aditiva del currículo según la cual los elementos curriculares no forman parte de un sistema de ideas integrado y coherente.

Respecto de la influencia del dominio de formación cuando las disciplinas son similares, no se encontraron diferencias significativas como así tampoco se evidenció influencia de las demás características académicas que se estudiaron.

Cabe destacar que los resultados se limitan a los docentes que se desempeñan en la Facultad de Ingeniería de la UNMdP, quedando pendiente para investigaciones futuras ampliar la muestra a otras universidades.

Por último, dado que las concepciones pueden entenderse como estados en desarrollo que pueden ser modificados comenzando por hacerlas explícitas, esta investigación pretende hacer un aporte también en el sentido de haber proporcionado una instancia de reflexión al exponer a los docentes a los dilemas, generando un espacio de discusión profesional que puede contribuir a resignificar la acción docente en el aula universitaria. Teniendo en cuenta que estas concepciones impactarán directamente en la formación recibida por el futuro profesional, a través de las decisiones tomadas por los docentes en el uso de dinámicas y estrategias en el desarrollo de sus clases, la reflexión sobre las mismas se hace trascendente a la hora de pensar la formación de los ingenieros del siglo XXI.

Experiencias como las de Cranton y Carusetta (2002) y la de Rodríguez-Sosa y HernándezSánchez (2018) han notado que el proceso de enfrentar a los docentes con sus propios supuestos sobre la enseñanza y, posteriormente, realizar un debate sobre estos supuestos, ha conducido a la redescripción de sus concepciones hacia visiones pedagógicas y prácticas más actuales. 


\section{Referencias}

Alves, I. \& Pozo, J. (2020). Las teorías implícitas de profesores universitarios brasileños acerca de la motivación de sus alumnos para aprender. Calidad en la Educación, (53), 252283. doi: 10.31619/caledu.n53.871

Borgovello, A., Sartori, M. \& Sanjurjo, L. O. (2018). Concepciones de docentes sobre los estudiantes y sus prácticas pedagógicas. Educación y Educadores, 21(1), 27-48. doi: 10.5294/edu.2018.21.1.2

Boud, D., Cohen, R. \& Sampson, J. (Eds.). (2014). Peer learning in higher education: Learning from and with each other. Londres, Reino Unido: Routledge.

Buffa, F. A., García, M. B., Moro, L. E., Menna, M., Massa, P., Fanovich, M. A. \& Fuchs, V. M. (2020). Concepciones acerca de la enseñanza en docentes de Ingeniería: diseño y validación de un cuestionario de dilemas y estudio descriptivo. Revista Educación en Ingeniería, 15(30), 18-25. doi: 10.26507/rei.v15n30.1088

Buffa, F., Massa, P., Moro, L. \& García, M. B. (2019). Concepciones de docentes de ingeniería acerca de la enseñanza. Análisis de la consistencia. Revista Educación en Ingeniería, 14(27), 89-96.

Buffa, F., Moro, L., Massa, P., García, M. B. \& Fanovich, M. A. (2018). Las concepciones sobre la enseñanza en docentes universitarios de Ingeniería. Revista Facultad de Ciencias Exactas Físicas y Naturales, 5, Suplemento 2, 123-130.

Claxton, G. (1990). Teaching to learn: A direction for education. Londres, Reino Unido: Weidenfeld \& Nicolson.

Consejo Federal de Decanos de Ingeniería. (2018). Libro Rojo de CONFEDI (2018). Propuesta de estándares de segunda generación para la acreditación de carreras de ingeniería en la república argentina. Recuperado desde https://confedi.org.ar/download/documentos confedi/LIBRO-ROJO-DE-CONFEDI-Estandares-de-Segunda-Generacionpara-Ingenieria-2018-VFPublicada.pdf 
ANÁLISIS DE LAS CONCEPCIONES DE DOCENTES SOBRE LA ENSEÑANZA DE LA INGENIERÍA A TRAVÉS

DE UN CUESTIONARIO DE DILEMAS Y ENTREVISTAS SEMIESTRUCTURADAS

Cranton, P. \& Carusetta, E. (2002). Reflecting on teaching: the influence of context. The International Journal for Academic Development, 7(2), 167-176. doi: 10.1080/1360144032000071288.

Cukierman, U. (2018). Aprendizaje centrado en el estudiante: un enfoque imprescindible para la educación en ingeniería. En L. A. González Araujo (Ed.), Aseguramiento de la calidad y mejora en la educación en ingeniería: Experiencias en América Latina (pp. 27-39). Bogotá, Colombia: ACOFI y CONFEDI.

Díaz, A. H. \& Hernández, M. G. (2015). Estrategias de aprendizaje en la formación universitaria. Educere, 19(63), 441-454.

Errázuriz-Cruz, M. (2020). Teorías implícitas sobre escritura de estudiantes y formadores de pedagogía. Magis, Revista Internacional de Investigación en Educación, 13, 1-26. doi: 10.11144/Javeriana.m13.tiee

Flórez Nisperuza, E. P., Páez, J., Fernández, C. M. \& Salgado, J. F. (2019). Reflexiones docentes acerca de las concepciones sobre la evaluación del aprendizaje y su influencia en las prácticas evaluativas. Revista Científica, 34(1), 63-72. doi: $10.14483 / 23448350.13553$

García, M. B. (2009). Las concepciones epistemológicas en docentes universitarios de ciencias (Tesis doctoral). Recuperado desde https://repositorio.uam.es/han-dle/104$\underline{86 / 131371}$

García, M. B., Martín, S. \& Mateos Sanz, M. (2018). Identificación de concepciones de profesores universitarios sobre la enseñanza y la evaluación. Revista Docencia Universitaria, 17, 17-42.

Husson, F., Lê, S. \& Pagès, J. (2017). Clustering. Exploratory multivariate analysis by example using $R, 173-208$.

Kowalski, V., Erck, I. \& Enríquez, H. (2018). Avances en el modelo operativo para el diseño de asignaturas orientadas a la formación por competencias en ingeniería. Universidad Nacional de Misiones. Recuperado desde https://docer.com.ar/doc/8xcvne 
Kramer, A. B. (2016). Las concepciones epistemológicas y didácticas explícitas, de alumnos del Profesorado de Biología, de un Instituto de Formación Docente de Corrientes, Argentina. Revista de Educación en Biología, 19(1), 125-127.

Mainer Baqué, J. (2019). La Profesionalización Docente En Perspectiva Crítico-Genealógica. El Caso Español. Revista Binacional Brasil Argentina Rbba, 8(2), 80-94.

Martín, S., Vilanova, S. \& García, M. (2013). Saber decir y saber hacer en la enseñanza de las ciencias: las representaciones sobre el aprendizaje y práctica en el aula en un grupo de docentes universitarios de Biología. Revista de Educación en Biología, 16(2), 3140.

Norton, L., Richardson, T. E., Hartley, J., Newstead, S. \& Mayes, J. (2005). Teachers' beliefs and intentions concerning teaching in higher education. Higher education, 50(4), 537571.

Oliver, M. I, Villalonga de García P., García, M. B., Natal, M. \& Chaparro, M. (2017). Concepciones de los docentes universitarios de matemática graduados y en formación: ¿qué relación hay entre ellas? Revista Docencia Universitaria, 18(1), 131150.

Pacheco Lora, L. C. (2019). Representaciones explícitas e implícitas sobre el aprendizaje y la enseñanza en una muestra de docentes del departamento de Córdoba-Colombia (Tesis doctoral). Recuperado desde https://manglar.uninorte.edu.co/handle/10584/8801\#page=1

Pecharromán, I. (2003). Teorías epistemológicas implícitas en diferentes dominios. Influencia de variables relacionadas con la instrucción. Madrid, España: Universidad Autónoma de Madrid.

Pérez Echeverría, M. P., Mateos, M., Pozo, J. I. \& Scheuer, N. (2001). En busca del constructivismo perdido: concepciones implícitas sobre el aprendizaje. Estudios de psicología, 22(2), 155-173.

Pozo, J. I., Loo, C. \& Martín, E. (2016). El cambio de las concepciones y las prácticas docentes como factor de cambio educativo. En J. Manzi \& R. M. García (Eds.), Abriendo las 
ANÁLISIS DE LAS CONCEPCIONES DE DOCENTES SOBRE LA ENSEÑANZA DE LA INGENIERÍA A TRAVÉS

DE UN CUESTIONARIO DE DILEMAS Y ENTREVISTAS SEMIESTRUCTURADAS

puertas del aula: nuevos enfoques para la transformación de las prácticas docentes (pp. 545-584). Santiago de Chile: Pontificia Universidad Católica.

Ramsden, P., Prosser, M., Trigwell, K. \& Martin, E. (2007). University teachers' experiences of academic leadership and their approaches to teaching. Learning and Instruction, $17(2), 140-155$

Ravanal, E., Camacho, J., Escobar, L. \& Jara, N. (2014). ¿Qué dicen los profesores universitarios de ciencias sobre el contenido, metodología y evaluación? Análisis desde la acción educativa. REDU. Revista de Docencia Universitaria, 12(1), 307-335.

Rodrigo, M. J., Rodríguez, A. \& Marrero, J. (1993). Las teorías implícitas. Una aproximación al conocimiento cotidiano. Madrid, España: Editorial Visor.

Rodríguez-Sosa, J. \& Hernández-Sánchez, K. (2018). Problematización de las prácticas docentes y contextualización de la enseñanza. Propósitos y Representaciones, 6(1), 507-541. doi: 10.20511/pyr2018.v6n1.211

Savasci-Acikalin, F. (2009). Teacher beliefs and practice in science education. Asia-Pacific Forum on Science Learning \& Teaching, 10(1), 1-14.

Schommer-Aikins, M., Duell, O. K. \& Barker, S. (2003). Epistemological beliefs across domains using Biglan's classification of academic disciplines. Research in Higher Education, 44, 347-366.

Schraw, G. (2013). Review Article Conceptual Integration and Measurement of Epistemological and Ontological Beliefs in Educational Research. Londres, Reino Unido: Hindawi Publishing Corporation. doi: 10.1155/2013/327680

Soler, M. G., Cárdenas, F. A. \& Hernández-Pina, F. (2018). Enfoques de enseñanza y enfoques de aprendizaje: perspectivas teóricas promisorias para el desarrollo de investigaciones en educación en ciencias. Ciênc. Educ., Bauru, 24(4), 993-1012.

Soler-Contreras, M. G., Cárdenas-Salgado, F. A., Hernández-Pina, F. \& Monroy-Hernández, F. (2017). Enfoques de aprendizaje y enfoques de enseñanza: origen y evolución. Educación y Educadores, 20(1), 65-88. doi: 10.5294/edu.2017.20.1.4 
ANÁLISIS DE LAS CONCEPCIONES DE DOCENTES SOBRE LA ENSEÑANZA DE LA INGENIERÍA A TRAVÉS DE UN CUESTIONARIO DE DILEMAS Y ENTREVISTAS SEMIESTRUCTURADAS

Ventura, A. C. (2016). ¿Enseño como aprendí?: el rol del estilo de aprendizaje en la enseñanza del profesorado universitario. Aula Abierta, 44(2) 91-98. doi: 10.1016/j.aula.2016.05.001

Vilanova, S. L., Mateos Sanz, M. D. M. \& García, M. B. (2011). Las concepciones sobre la enseñanza y el aprendizaje en docentes universitarios de ciencias. Revista iberoamericana de educación superior, 2(3), 53-75. 